\title{
Macroprolactinaemia: a biological diagnostic strategy from the study of 222 patients
}

\author{
Lucile Parlant-Pinet ${ }^{1}$, Catherine Harthé ${ }^{2}$, Florence Roucher ${ }^{1,2}$, Yves Morel ${ }^{1,2}$, \\ Françoise Borson-Chazot ${ }^{1,3}$, Gérald Raverot ${ }^{1,3}$ and Véronique Raverot ${ }^{2}$ \\ ${ }^{1}$ Faculté de Médecine Lyon-Est, Université Claude Bernard Lyon 1, Lyon, France, ${ }^{2}$ Hospices Civils de Lyon, \\ Laboratoire d'Hormonologie, Centre de Biologie et de Pathologie Est, and ${ }^{3}$ Hospices Civils de Lyon, \\ Fédération d'endocrinologie, Groupement Hospitalier Est, 59 bd Pinel, F-69677 Bron Cedex, France
}

Correspondence should be addressed to V Raverot Email veronique.raverot@ chu-lyon.fr

\begin{abstract}
Objectives: Gel filtration chromatography (GFC), the gold standard for macroprolactinaemia (MPRL) diagnosis, is a slow, costly and labour-intensive method. To limit the number of GFC required, we evaluated two screening tests for MPRL: prolactin (PRL) recovery after polyethylene glycol (PEG) precipitation and PRL concentration ratio, derived from two assays, each having different big-big-PRL cross-reactivities.

In some patients, MPRL is characterised by clinical symptoms which can be associated with an excess of monomeric PRL. We compared the monomeric PRL concentration obtained from GFC with the PRL concentration i) on a cobas e 601 analyser and ii) in the supernatant after PEG precipitation.

Design and methods: We studied hyperprolactinaemic sera subjected to physician-ordered GFC, between February 2013 and July 2014. We performed PEG precipitation (to evaluate the PRL concentration and rate of recovery in the supernatant) and two PRL assays: RIA and electrochemiluminescent assay (ECLIA), on a Roche cobas e 601 analyser, and calculated the RIA/ECLIA ratio.

Results: Among the 222 sera, we were able to diagnose or exclude MPRL in $72.1 \%$ of cases, based solely on the ratio and/or recovery. In the remaining cases, GFC was necessary for making a diagnosis. Elevated monomeric PRL was present in $10.9 \%$ of macroprolactinaemic sera. In the case of MPRL, both PRL measurements on the cobas analyser and in the supernatant weakly correlated with monomeric PRL values obtained from GFC.

Conclusions: The combination of PEG and RIA/ECLIA ratio analysis reduced the number of necessary GFC. However, GFC is essential in MPRL cases to evaluate the monomeric PRL concentration.

European Journal of

Endocrinology

(2015) 172, 687-695
\end{abstract}

\section{Introduction}

Three different molecular forms of circulating prolactin (PRL) have been described, categorised according to their molecular mass: monomeric PRL (23 kDa), big-PRL (bPRL, 40-60 kDa) and big-big-PRL (bbPRL, $>100 \mathrm{kDa}$, also called macroprolactin). Monomeric PRL is generally the most common form circulating in healthy subjects and in hyperprolactinaemic sera $(1,2)$. Macroprolactinaemia (MPRL) is the condition which results when the high molecular mass forms (bPRL, bbPRL) are predominant. MPRL is classically associated with asymptomatic
(C) 2015 European Society of Endocrinology Printed in Great Britain hyperprolactinaemia $(3,4)$, though symptoms of hyperprolactinaemia have been described in some macroprolactinaemic patients $(5,6,7,8,9)$. The prevalence of MPRL has been estimated at being between 10 and $45 \%$ in the hyperprolactinaemic population $(2,5,6,10)$. In most cases, bbPRL is composed of immune complexes of PRL and anti-PRL auto-antibodies $(\operatorname{IgG})(7,11,12,13)$. The subsequent hyperprolactinaemia may be attributed to a diminished clearance rate, due to the high molecular size of these bbPRL complexes (11). Detection of bbPRL is

Published by Bioscientifica Ltd. 
commonly undertaken by PRL immunoassays, with variable, system-dependent cross-reactivities $(14,15,16)$. However, the anti-PRL auto-antibodies can mask the immunoassay epitopes of interest and/or interfere with binding to the solid phase, to various degrees (15).

As MPRL can lead to delayed diagnosis or even misdiagnosis and inappropriate treatment, it has been suggested that all hyperprolactinaemic sera should be routinely screened for bbPRL $(8,17,18,19)$. Although bbPRL may be the predominant molecular form in MPRL, monomeric PRL may simultaneously be found in excess. Therefore, MPRL could be associated with monomeric hyperprolactinaemia, which may lead to the development of hyperprolactinaemic symptoms. Thus, in the case of MPRL, it is important to also determine the monomeric PRL concentration (8). Gel filtration chromatography (GFC) is the gold standard method for diagnosing MPRL and quantifying PRL molecular forms in sera, including monomeric PRL, but this is a slow, costly and labourintensive method. In order to limit the number of GFC required, alternative separation methods have been developed. Amongst them, polyethylene glycol (PEG) precipitation of bbPRL is the most commonly employed $(19,20,21,22)$. Analysis of the discrepancies between results obtained from two PRL assays, with different crossreactivities, has been suggested as a viable MPRL analysis technique $(23,24)$. We evaluated two MPRL screening methods: first, the rate of PRL recovery in the supernatant after PEG precipitation and secondly, the ratio of PRL concentrations derived from two different assays (RIA and electrochemiluminescence assay with Prolactin II on a Roche cobas e 601 analyser). Furthermore, to assess alternative approaches for monomeric PRL quantification, PRL measurement with a low-reacting assay (Prolactin II assay on a Roche cobas e 601 analyser) and PRL measurement in the supernatant after PEG precipitation $(1,22,25,26)$ were performed.

\section{Subjects and methods}

\section{Study patients}

From February 2013 to July 2014, 624 sera samples, with accompanying medical prescriptions requesting PRL GFC, were received at the Centre de Biologie et Pathologie Est (Bron, France). Hyperprolactinaemia was confirmed (with RIA and/or electrochemiluminescent assay (ECLIA)) in 386 of these samples. Of these 386 hyperprolactinaemic sera, we further analysed 222 randomly selected samples. Each sample was divided into two different aliquots.
ECLIA measurements were performed on the first aliquots immediately, while the second aliquots were stored at $-20^{\circ} \mathrm{C}$ until RIA analysis. The RIA measurements before and after PEG precipitation were performed on the same day once a week. The GFC was performed, when required, on the same aliquot, after one more freeze/thaw cycle. The samples which were drawn in our institution were split and frozen on the day of collection. The samples originating from other hospitals or laboratories were already frozen upon arrival at our institution. These were assayed after two freeze/thaw cycles.

\section{Assay methods}

Electrochemiluminescent assay $\checkmark$ The concentrations of PRL were measured on a cobas e 601 analyser with Prolactin II reagent (Roche Diagnostics), using an immunochemical method based on the sandwich principle, with two monoclonal PRL-specific antibodies. One biotinylated antibody interacted with streptavidincoated micro particles. The secondary antibody was labelled with a ruthenium complex. After application of a voltage, chemiluminescent emissions were assayed. This assay has a low cross-reactivity towards bbPRL. The reference range was $4-15.2 \mu \mathrm{g} / \mathrm{l}$ for men and $4.8-23.3 \mu \mathrm{g} / \mathrm{l}$ for women. Internal controls on two levels were run each day (PCU1 and PCU2, Roche). The interassay coefficient of variation $(\mathrm{CV})$ was $3.77 \%$ at level $11.5 \mu \mathrm{g} / \mathrm{l}$ and $4.01 \%$ at level $42.2 \mu \mathrm{g} / \mathrm{l}$.

RIA $>$ Our in-house RIA is based on the competition principle. The serum samples were incubated for $18-24 \mathrm{~h}$ at $5 \pm 3{ }^{\circ} \mathrm{C}$ with PRL labelled with ${ }^{125} \mathrm{I}(20000 \mathrm{cpm} /$ sample) and in-house rabbit anti-human PRL antibodies (27). These polyclonal antibodies were non-specific for the monomeric form of PRL (28). Antigen-antibody complexes were separated from free PRL by precipitation using a precipitating reagent (Institut National de la Recherche Agronomique). The precipitate was washed with gelatin phosphate buffer and the radioactivity in the remaining pellet was measured with a $\gamma$-counter. Duplicate analyses were performed on each serum. Our RIA method detects all molecular forms of PRL. The reference range was $<25 \mu \mathrm{g} / \mathrm{l}$ for men and $<30 \mu \mathrm{g} / \mathrm{l}$ for women. The inter-assay CV was $8.0 \%$ at level $17.85 \mu \mathrm{g} / \mathrm{l}$ and $8.5 \%$ at level $60.93 \mu \mathrm{g} / \mathrm{l}$.

For both methods (ECLIA and RIA), calibrators were traceable to the WHO Third International Standard $84 / 500$. The PRL concentrations in $\mu \mathrm{g} / \mathrm{l}$ can be converted to $\mathrm{mIU} / \mathrm{l}$ by multiplying by 21.2 . 
We calculated the PRL ratio in native sera according to the formula: PRL concentration derived from RIA $(\mu \mathrm{g} / \mathrm{l}) / \mathrm{PRL}$ concentration derived from ECLIA $(\mu \mathrm{g} / \mathrm{l})$.

\section{Separation methods}

PEG precipitation - The PEG precipitation was performed according to the general principle described by Fahie-Wilson \& Soule (2). A PEG solution was prepared using 25\% w/v PEG 6000 (Merck Schuchadt OHG), dissolved in freshly prepared PBS $\left(\mathrm{KH}_{2} \mathrm{PO}_{4}: 0.2 \mathrm{~g} / \mathrm{l}\right.$, $\left.\mathrm{Na}_{2} \mathrm{HPO}_{4}, 2 \mathrm{H}_{2} \mathrm{O}: 1.78 \mathrm{~g} / \mathrm{l}, \mathrm{KCl}: 0.2 \mathrm{~g} / \mathrm{l}, \mathrm{NaCl}: 8 \mathrm{~g} / \mathrm{l}\right)$ at room temperature. PEG $(100 \mu \mathrm{l})$ was added to $100 \mu \mathrm{l}$ of patients' serum and mixed by a vortex. The samples were incubated for $10 \mathrm{~min}$ at room temperature, and then were centrifuged at $4500 \boldsymbol{g}$ for $15 \mathrm{~min}$ at $4{ }^{\circ} \mathrm{C}$. PRL calibration standards, in plasma free PRL, were treated identically with PEG. The PRL concentrations were measured in $50 \mu \mathrm{l}$ of the supernatant using RIA. Hyperprolactinaemic sera with predominant monomeric PRL were used as internal controls. The inter-assay CV was $8.2 \%$ at level $60.6 \mu \mathrm{g} / \mathrm{l}$. The intra-assay CV was $4.8 \%$ at level $39.2 \mu \mathrm{g} / \mathrm{l}$. The interassay and intra-assay $\mathrm{CV}$ for macroprolactinaemic serum were 12.1 and $8.6 \%$ respectively (at level $14.9 \mu \mathrm{g} / \mathrm{l}$ ). The PRL recovery was calculated according to the formula: (PRL in the supernatant after PEG precipitation, yielded from RIA analysis $(\mu \mathrm{g} / \mathrm{l}) / \mathrm{PRL}$ in the native serum yielded from RIA analysis $(\mu \mathrm{g} / \mathrm{l})) \times 100$.

To establish an upper limit of PRL concentration in the supernatant after PEG precipitation in female samples, we tested sera from 46 normoprolactinaemic women (normal PRL concentrations obtained by RIA and ECLIA analyses).

Gel filtration chromatography $>$ All GFC analyses were performed at room temperature with PBS on a Sephacryl $200 \mathrm{HR}(45 \times 1.5 \mathrm{~cm})$ column. Before analysis, the column was calibrated with molecular weight markers: Dextran Blue (2000 kDa), monomeric PRL (23 kDa) labelled with ${ }^{125} \mathrm{I}(10000 \mathrm{cpm}, 20 \mu \mathrm{l})$ and ovalbumin (45 kDa) labelled with ${ }^{125} \mathrm{I}(10000 \mathrm{cpm}, 20 \mu \mathrm{l})$. One millilitre of plasma (or diluted plasma if the concentration was $>200 \mu \mathrm{g} / \mathrm{l}$, in order to obtain a PRL concentration of around $200 \mu \mathrm{g} / \mathrm{l}$ ), together with Dextran Blue and a tracer dose of ${ }^{125}$ I-PRL, was applied to the column. Elution was carried out using PBS, at a flow rate of $0.4 \mathrm{ml} / \mathrm{min}$. The fractions were collected every $4 \mathrm{~min}$. Radioactivity was measured with a $\gamma$-counter. Monomeric PRL ( $23 \mathrm{kDa})$ was eluted in fractions which corresponded to the ${ }^{125}$ I-PRL-induced peak of radioactivity. The two previous fractions may also have contained glycosylated monomeric PRL (25-29 kDa).
Dextran Blue allowed the estimation of the exclusion volume of the column and the elution fractions for bbPRL. The fractions were then pooled, two-by-two, and $400 \mu \mathrm{l}$ were taken from each pooled eluate and analysed for PRL using RIA. The relative amounts of monomeric PRL, bPRL and bbPRL in each serum were determined from the GFC elution profile.

The monomeric PRL concentration after GFC (GFC monomeric PRL) was calculated according to the formula: (amount of monomeric PRL from GFC $(\%) / 100) \times P R L$ concentration in the native serum obtained by RIA $(\mu \mathrm{g} / \mathrm{l})$. We considered that normal monomeric PRL values obtained from GFC for women were $\leq 21 \mu \mathrm{g} / \mathrm{l}$ ( $\leq 444 \mathrm{mUI} / \mathrm{l})$, as reported by Beltran et al. (1).

\section{Definition of groups}

The samples were classified according to the molecular PRL form which was present in the highest proportion in the eluate, after GFC. The samples with predominantly bbPRL or bPRL were classified as part of the MPRL group. The samples with predominantly monomeric PRL made up the monomeric PRL predominance (mPRL) group.

\section{Statistical analyses}

Statistical analyses were performed using GraphPad Prism version 5.01. A two-tailed $P$ value of $<0.05$ was considered to be statistically significant. We assessed the differences in categorical variables with the $\chi^{2}$ test. The medians were compared with the Mann-Whitney $U$ (unpaired data) and Wilcoxon (paired data) tests. The associations between continuous variables were evaluated with the nonparametric Spearman's rank correlation coefficient. Comparisons to a reference method were assessed using Deming regression (slope and Y-intercept). We assessed agreement with Bland-Altman analysis.

\section{Results}

\section{Study population characteristics}

We studied 222 sera obtained from 197 women (age range 10-78 years) and 25 men (age range 17-76 years). The median PRL concentrations calculated by RIA and ECLIA were 47.4 and $49.5 \mu \mathrm{g} / \mathrm{l}$ for women, and 38.4 and $36.7 \mu \mathrm{g} / \mathrm{l}$ for men respectively.

The MPRL group contained 64 samples (28.8\% of the study population). In 63 cases, 54 women (age range 15-74 
Table 1 Characteristics of patients in both macroprolactinaemia (MPRL) and monomeric PRL predominance (mPRL) groups. The values are displayed as the median (minimum; maximum). The number of men and women in both groups were compared using the $\chi^{2}$ test. Medians were compared by the Mann-Whitney $U$ test. PRL recovery $(\%)=(P R L$ in the supernatant $(\mu g / l) / P R L$ in the native serum derived from RIA $(\mu \mathrm{g} / \mathrm{l})) \times 100$.

\begin{tabular}{l}
\hline Characteristics \\
\hline Demographics \\
Women $(n)$ \\
Men $(n)$ \\
Age (years) \\
GFC \\
Big-big-PRL $(\%)$ \\
Big-PRL $(\%)$ \\
Monomeric PRL $(\%)$ \\
Monomeric PRL $(\mu \mathrm{g} / \mathrm{l})$ \\
PRL assays (in native sera) \\
RIA ( $\mu$ g/l) \\
ECLIA ( $\mu$ g/l) \\
RIA/ECLIA \\
PEG precipitation \\
PRL in supernatant $(\mu g / l)$ \\
PRL recovery $(\%)$
\end{tabular}

\begin{tabular}{c}
\hline MPRL $(n=64)$ \\
55 \\
9 \\
$32.5(15 ; 76)$ \\
$78(24 ; 98)$ \\
$10(1 ; 54)$ \\
$12(1 ; 38)$ \\
$8.25(1.3 ; 64.6)$ \\
$72.45(21.5 ; 617.7)$ \\
$33.35(9.3 ; 156.6)$ \\
$2.035(0.64 ; 11.16)$ \\
\\
$12.75(3.3 ; 136.4)$ \\
$19.95(2.1 ; 77.9)$
\end{tabular}

\begin{tabular}{c}
\hline mPRL $(n=158)$ \\
142 \\
16 \\
$34(10 ; 78)$ \\
$16(3 ; 40)$ \\
$11(1 ; 31)$ \\
$71(40 ; 96)$ \\
$28.5(10.7 ; 431.7)$ \\
$39.95(17.7 ; 449.7)$ \\
$53.35(21.8 ; 523.3)$ \\
$0.75(0.55 ; 1.83)$ \\
$32.85(10.6 ; 641.9)$ \\
$76.45(31.8 ; 142.7)$
\end{tabular}

\begin{aligned} \hline $\boldsymbol{P}$ values \\ \\ \\ 0.4008 \\ 0.4008 \\ 0.6083 \\ \\ $<0.0001 \\ 0.1356 \\ <0.0001 \\ <0.0001 \\ \\ <0.0001 \\ <0.0001 \\ <0.0001 \\ <0.0001 \\ <0.0001\end{aligned}$

A $P$ value of $<0.05$ is significant. GFC, gel filtration chromatography; PRL, prolactin; ECLIA, electrochemiluminescent assay; PEG, polyethylene glycol.

years) and nine men (age range 21-76 years), bbPRL was the predominant form. Only one woman had a predominant bPRL form (21\% monomeric PRL, 54\% bPRL, 24\% bbPRL). Patient characteristics for both the MPRL and mPRL groups are given in Table 1.

The PRL concentration obtained from RIA and ECLIA assays were closely correlated in the MPRL group $(r=0.909, P<0.0001)$. This correlation was lower in the MPRL group $(r=0.598, P<0.0001)$.

\section{Screening for MPRL}

PRL recovery after PEG precipitation - The PRL recovery rates for both groups are given in Table 1 . The receiver operating characteristic (ROC) curve, for recovery against GFC, had an AUC of $0.9739(P<0.0001,95 \%$ CI: $0.9501-$ 0.9978). Thus, the PRL recovery method was highly effective for the identification of MPRL.

A recovery value of $<30 \%$ ( $19.8 \%$ of the samples) was $100 \%$ specific for detecting MPRL. The values of $>80 \%$ (31.1\% of the samples) indicated an absence of MPRL. Recovery rates for half of the samples (49.1\%) were in a grey area (between 30 and 80\%), and GFC was necessary for successful diagnosis. GFC showed MPRL in $18.3 \%$ of these latter cases (Fig. 1). According to the ROC curve for recovery against GFC, a recovery value of $<65 \%$ ( $48.6 \%$ of the samples) indicated MPRL with a sensitivity of $96.9 \%$ and a specificity of $70.9 \%$.
RIA/ECLIA PRL ratios $~$ In the MPRL group, PRL concentrations yielded from RIA were higher than those from ECLIA (cf Table $1, P<0.0001$ ). The RIA/ECLIA PRL ratios for each group are given in Table 1 . The ROC curve for the PRL ratio against GFC had an AUC of 0.9683 (95\% CI: 0.9381-0.9984), which demonstrates the high

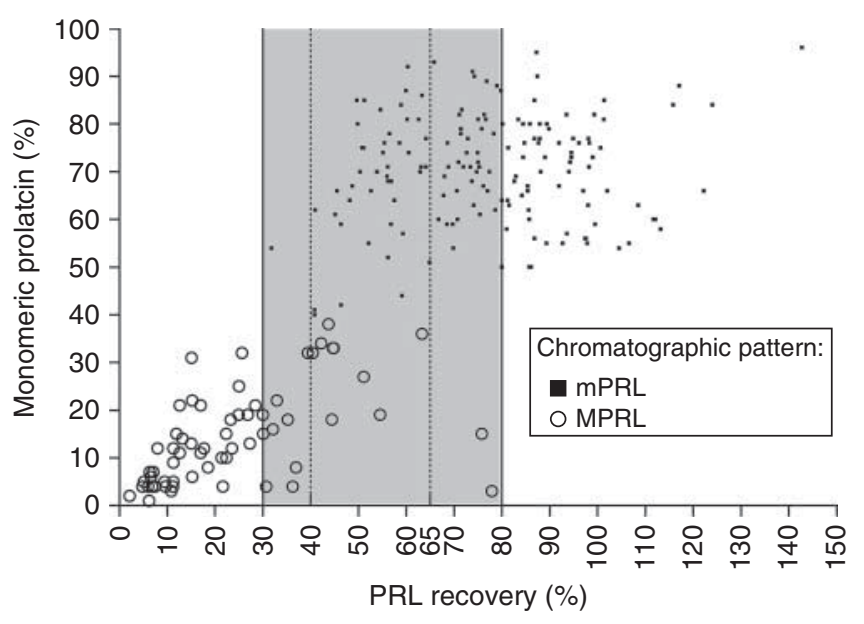

\section{Figure 1}

Percentage monomeric prolactin (PRL) after gel filtration chromatography and PRL recovery after polyethylene glycol precipitation, according to the chromatographic pattern (mPRL, monomeric PRL predominance; MPRL, macroprolactinaemia). 


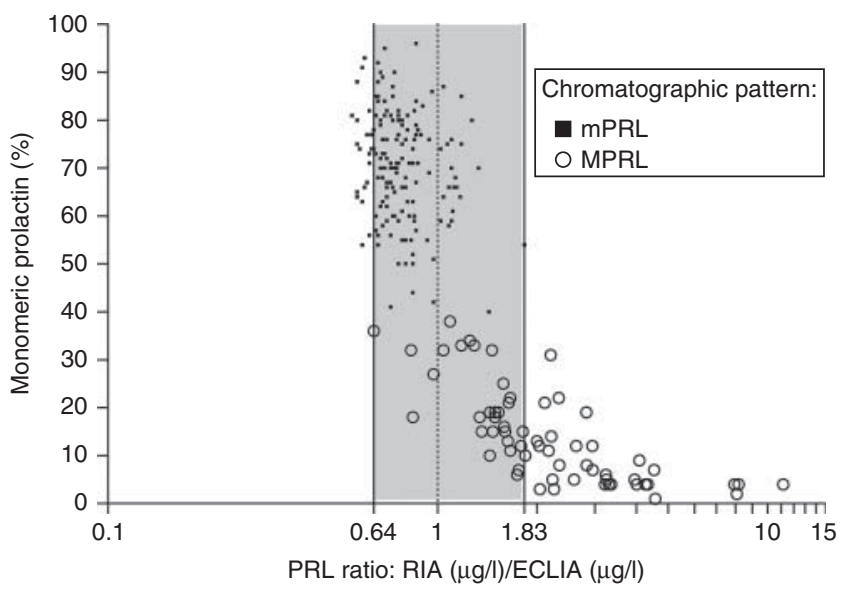

\section{Figure 2}

Percentage monomeric prolactin (PRL) after gel filtration chromatography and RIA/ECLIA PRL ratio, according to the chromatographic pattern (mPRL, monomeric PRL predominance; MPRL, macroprolactinaemia).

performance level of this technique for the identification of MPRL.

Samples presenting a ratio of $>1.83(15.8 \%)$ were all macroprolactinaemic samples. All samples presenting a ratio of $<0.64(9.4 \%)$ contained predominant monomeric PRL. The remaining $74.8 \%$ of samples fell between these two groups, with ratios of between 0.64 and 1.83 . GFC showed that among these latter samples, $17.5 \%$ were macroprolactinaemic (Fig. 2). According to the ROC curve for the PRL ratio against GFC, a ratio value of $>1$ (36.5\% of the samples) indicated MPRL with a sensitivity of $93.8 \%$ and a specificity of $86.7 \%$.

Combination of PEG precipitation and RIA/ECLIA PRL ratio $>$ The PRL ratios and recovery rates are presented in Fig. 3. All samples with a ratio of $<1$ and recovery of $>65 \%$ (48.6\% of the samples) contained predominant monomeric PRL, according to GFC analysis. For these samples, the recovery rate together with the ratio could exclude MPRL diagnosis. All samples with a ratio of $<0.64$ and/or a recovery of $>80 \%$ (33.3\% of the samples) contained predominant monomeric PRL. All samples with a ratio of $>1.83$ and/or a recovery of $<30 \%(22.1 \%$ of the samples) were macroprolactinaemic. Some samples satisfied several of these criteria.

GFC was necessary for the samples that did not meet with one or more of these criteria (27.9\% of the samples). GFC showed MPRL in $24.2 \%$ of these latter cases. We could ultimately diagnose MPRL, with a sensitivity of $100 \%$.

\section{Evaluation of monomeric PRL concentration}

GFC monomeric PRL - The GFC monomeric PRL values for both groups are given in Table 1. In the MPRL group, seven female patients (10.9\%) had a GFC monomeric PRL value of above $21 \mu \mathrm{g} / \mathrm{l}(24.2-64.6 \mu \mathrm{g} / \mathrm{l})$. These seven patients had an excess of monomeric PRL, associated with MPRL.

PRL concentrations derived from ECLIA The PRL concentrations obtained by ECLIA (Table 1) correlated strongly with GFC monomeric PRL values. However, the correlation was lower in the MPRL group than in the mPRL group: $r=0.687(P<0.0001)$ vs $r=0.906, P<0.0001$ (Fig. 4). In the MPRL group, the slope and Y-intercept within a 95\% CI were $(2.779 ; 4.526 \mu \mathrm{g} / \mathrm{l})$ and $(-11.53$; $14.97 \mu \mathrm{g} / \mathrm{l})$.

We evaluated the bias for PRL values obtained by ECLIA, compared with GFC monomeric PRL: \% difference $(100 \times($ PRL from ECLIA - GFC monomeric PRL)/average $)$ vs average ((PRL from ECLIA + GFC monomeric PRL)/2). In the MPRL group, the bias (95\% limits of agreement) was $+119.24 \%$ (+59.76\%; +178.72\%) (Fig. 5).

PRL in the supernatant after PEG precipitation $>$ The PRL concentrations in the supernatants after PEG precipitation (Table 1) were strongly correlated with GFC monomeric PRL values in the $\mathrm{mPRL}$ group: $r=0.848$ $(P<0.0001)$. However, the correlation was lower in the

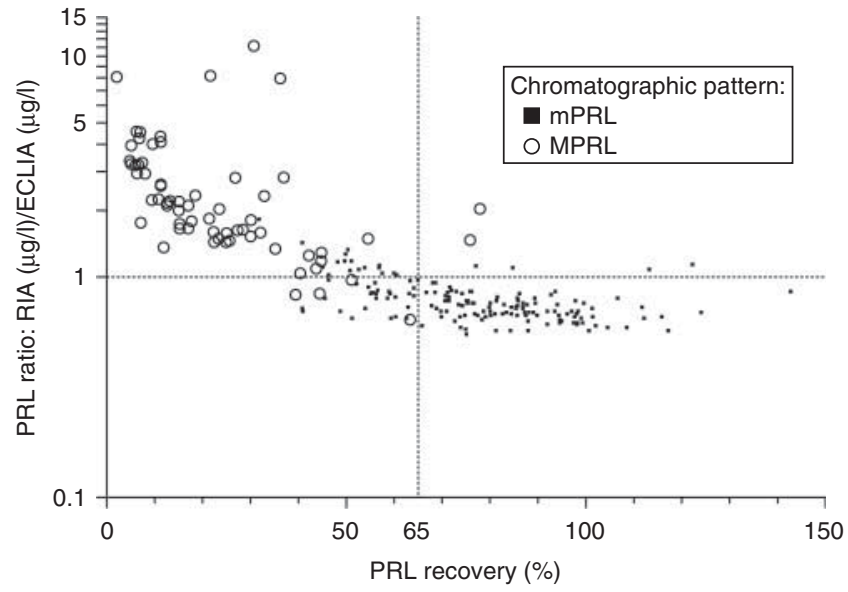

\section{Figure 3}

RIA/ECLIA prolactin (PRL) ratio and PRL recovery after polyethylene glycol precipitation, according to the chromatographic pattern (mPRL, monomeric PRL predominance; MPRL, macroprolactinaemia). 


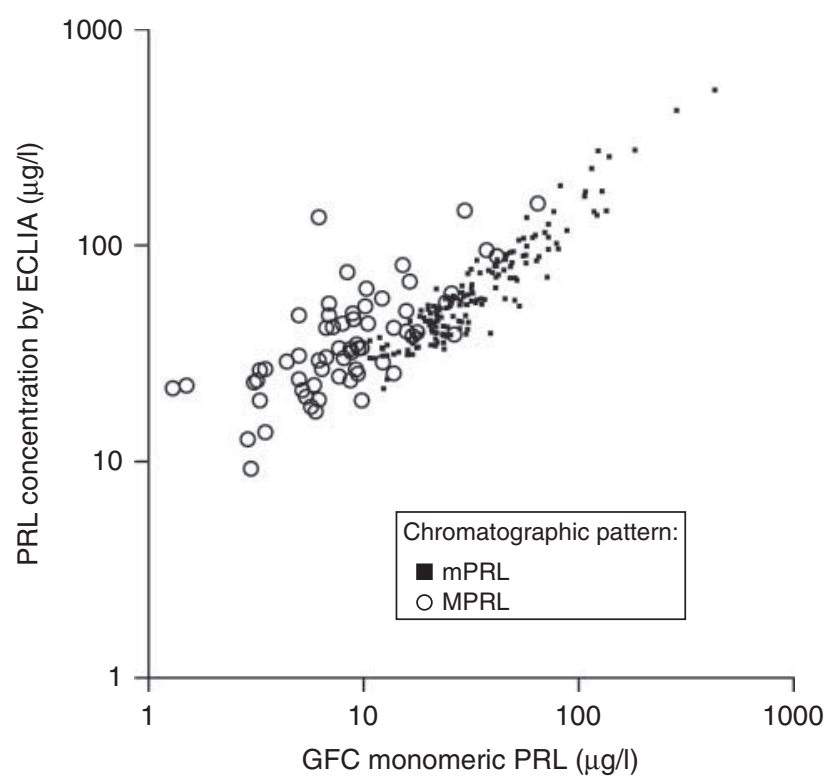

\section{Figure 4}

Prolactin (PRL) concentration by ECLIA and monomeric PRL concentration after gel filtration chromatography (GFC), according to the chromatographic pattern (mPRL, monomeric PRL predominance; MPRL, macroprolactinaemia). GFC monomeric $\mathrm{PRL}=$ (amount of monomeric PRL obtained by GFC (\%)/100) $\times$ PRL concentration in the native serum derived from RIA ( $\mu \mathrm{g} / \mathrm{l})$.

MPRL group: $r=0.561(P<0.0001)$ (Fig. 6). In the MPRL group, the slope and Y-intercept at 95\% CI were (1.716; $4.415 \mu \mathrm{g} / \mathrm{l})$ and $(-35.71 ; 5.223 \mu \mathrm{g} / \mathrm{l})$.

We evaluated the bias for PRL in the supernatant compared with GFC monomeric PRL: \% difference $(100 \times$ (PRL in the supernatant-GFC monomeric PRL)/average) vs average ((PRL in the supernatant $+\mathrm{GFC}$ monomeric PRL)/2). In the MPRL group, the bias was $+44.28 \%$ (-59.94\%; +148.50\%) (Fig. 7).

In the 46 normoprolactinaemic females, the PRL concentration in the supernatant had a normal distribution. This concentration was below $15 \mu \mathrm{g} / \mathrm{l}$ in $98 \%$ of these cases.

Of the 55 women of the MPRL group, 22 had elevated PRL concentrations in the supernatant $(>15 \mu \mathrm{g} / \mathrm{l})$. Six also had elevated GFC monomeric PRL $(>21 \mu \mathrm{g} / \mathrm{l})$. The remaining 16 women had normal GFC monomeric PRL $(<21 \mu \mathrm{g} / \mathrm{l})$, while PRL concentrations in the supernatant were elevated to between 15.4 and $136.4 \mu \mathrm{g} / \mathrm{l}$ (with six values above $30 \mu \mathrm{g} / \mathrm{l}$ ). In these latter cases, we could have misdiagnosed an excess of monomeric PRL, by assessing the PRL concentration in the supernatant alone.
Conversely, one woman had a normal supernatant PRL concentration $(12.9 \mu \mathrm{g} / \mathrm{l})$, while the GFC monomeric PRL was elevated $(26.4 \mu \mathrm{g} / \mathrm{l})$.

Taken together, 17 (30.9\%) of the 55 macroprolactinaemic women would have been misclassified based on assessment of the PRL concentration in the supernatant alone.

\section{Discussion}

We found MPRL to be prevalent in $28.8 \%$ of our 222 hyperprolactinaemic samples, an incidence which was close to that reported by Leslie et al. (6) (26.3\% in 1225 patients). These findings confirm that MPRL is a common condition. Moreover, we diagnosed MPRL by GFC, which remains the gold standard screening method. It should be noted that in this study, we examined a select population of hyperprolactinaemic patients in whom MPRL was suspected, as they were prescribed GFC.

Correct MPRL diagnosis is important to avoid unnecessary tests, misdiagnosis and inappropriate treatments in some cases of hyperprolactinaemia. We also chose to perform a high cross-reacting assay (RIA), in order to diagnose the largest possible number of existing MPRL, even in those with a normal PRL concentration, as demonstrated by a low cross-reacting assay such as

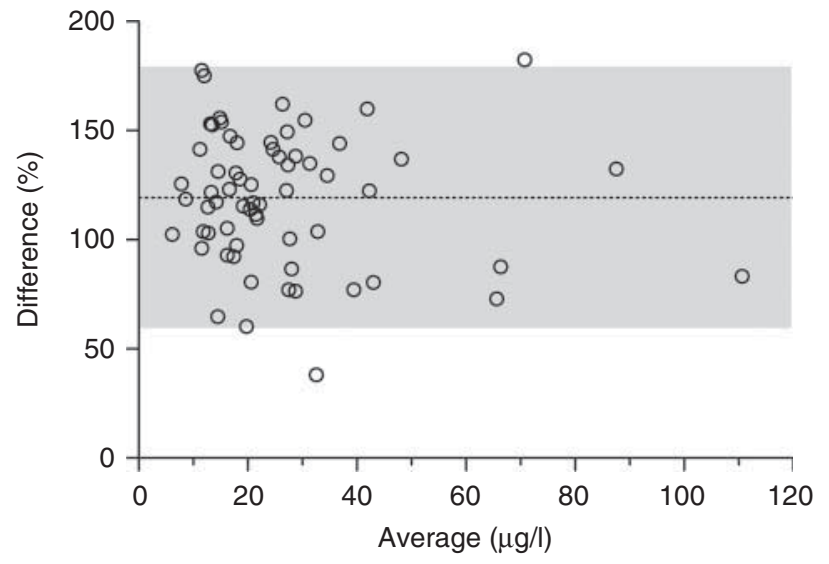

\section{Figure 5}

Bias for prolactin (PRL) concentration by ECLIA, compared with monomeric PRL concentration after gel filtration chromatography (GFC monomeric $\mathrm{PRL}$ ) in the macroprolactinaemia group: $\%$ difference $((100 \times$ (PRL from ECLIA - GFC monomeric $\mathrm{PRL})$ /average) vs average ((PRL from ECLIA + GFC monomeric $\mathrm{PRL}$ )/2). GFC monomeric PRL= (amount of monomeric PRL obtained by GFC (\%)/100) $\times$ PRL concentration in the native serum derived from RIA $(\mu \mathrm{g} / \mathrm{l})$. 


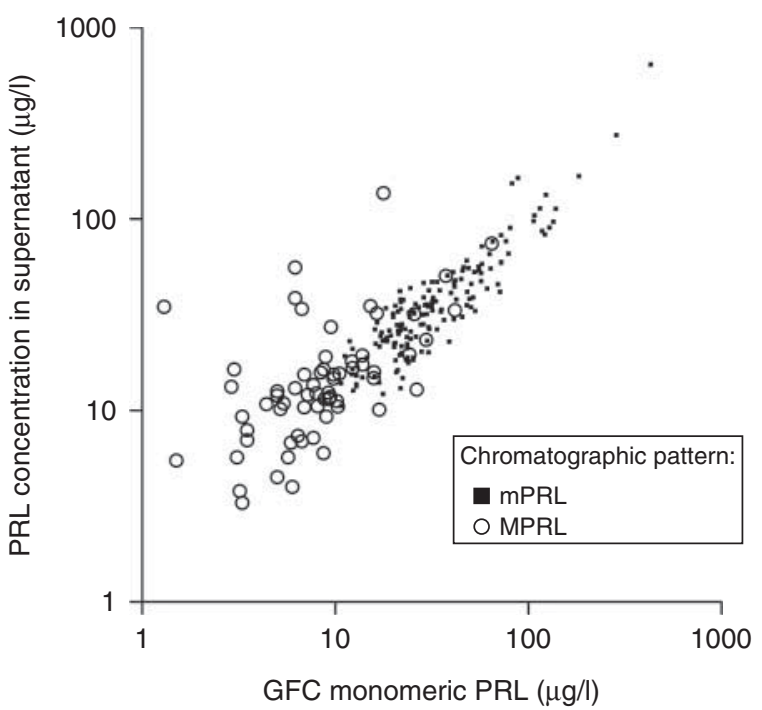

\section{Figure 6}

Prolactin (PRL) concentration in the supernatant after polyethylene glycol precipitation and monomeric PRL concentration after gel filtration chromatography (GFC), according to the chromatographic pattern (mPRL, monomeric PRL predominance; MPRL, macroprolactinaemia). GFC monomeric PRL = (amount of monomeric PRL yielded from GFC (\%)/100) $\times$ PRL concentration in the native serum from RIA $(\mu \mathrm{g} / \mathrm{l})$.

ECLIA. Indeed, ECLIA analysis showed that 14 women from our macroprolactinaemic population had normal PRL concentrations. Without a complementary high cross-reacting PRL assay, these 14 samples would not have been further analysed and the definitive diagnosis of MPRL would have been missed. We recognise that this RIA technique is not affordable in every laboratory, but as a reference centre it is essential that we do not miss an MPRL diagnosis, which is why we chose to continue to use it.

Given its high intrinsic performance (according to its ROC curve), PEG precipitation appeared to be a successful screening test for the diagnosis of MPRL. However, in our experience, the grey area of PRL recovery (30-80\%), for which GFC was necessary for diagnosis, was more extensive than that described previously $(19,20,21,29)$ and encompassed about half of the samples. On the basis of data shown in Fig. 1, we would have missed $17.2 \%$ of the MPRL cases, by using the classic cut-off of $40 \%$ recovery. Two sera with predominant bbPRL (95 and $78 \%$ of bbPRL) had particularly high PRL recovery rates (77.9 and $75.8 \%$ respectively). Heterogeneity of bbPRL could explain these results. For example, a small proportion of bbPRL is not in an IgG-bound form, but forms
IgA-PRL complexes, the precipitation of which may be incomplete (14).

In the MPRL group, the PRL established by RIA was almost always higher than the PRL by ECLIA. This is consistent with the higher cross-reactivity of RIA towards high molecular forms of PRL. This observation led us to use the ratio of the two values to screen for MPRL. Unlike PEG precipitation, this method has not yet been validated, though analysis of its ROC curve showed comparable intrinsic performances $(\mathrm{AUC}=0.9683$ vs 0.9739 for $\mathrm{PEG}$ precipitation) for the diagnosis of MPRL. However, with this method, about $75 \%$ of the samples fell into the grey area. If a high ratio $(>1.83)$ enabled us to confirm MPRL, lower values were less discriminate.

In our laboratory, in order to improve sensitivity, we chose to take into account the results of not only PRL recovery, but also the RIA/ECLIA PRL ratio. The results of the RIA/ECLIA ratio (if $>1.83$ or $<0.64$ ) and the PRL recovery (if $<30 \%$ or $>80 \%$ ), taken individually, allowed us to diagnose or exclude MPRL in $55.4 \%$ of the samples. Moreover, a combined criteria of RIA/ECLIA of $<1$ and PRL recovery of $>65 \%$ was achieved in $48.6 \%$ of our cohort and excluded MPRL. In all, we could provide a diagnosis without GFC for $72.1 \%$ of the hyperprolactinaemic samples. However, for the remaining $27.9 \%$ of the

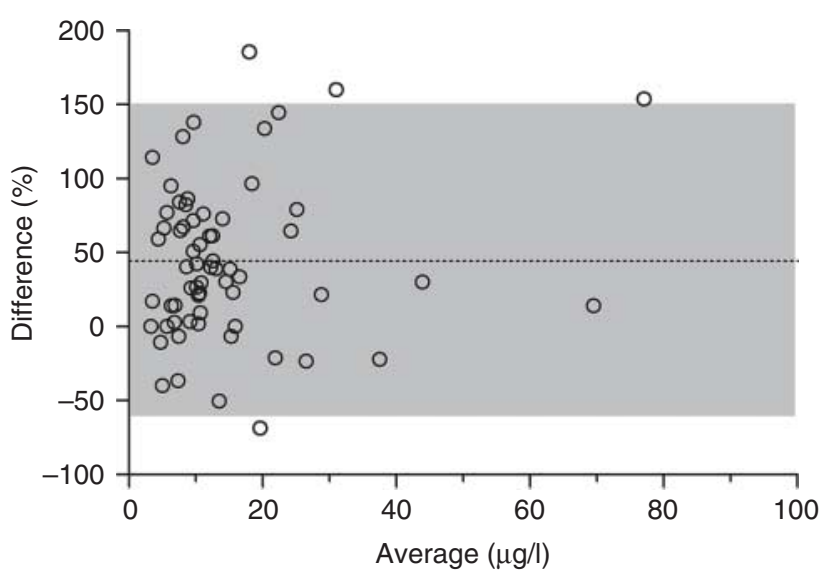

\section{Figure 7}

Bias for prolactin (PRL) concentration in the supernatant, compared with monomeric PRL concentration after gel filtration chromatography (GFC monomeric PRL) in the macroprolactinaemia group: $\%$ difference $((100 \times(P R L$ in the supernatant-GFC monomeric PRL)/average) vs average ((PRL in the supernatant $+\mathrm{GFC}$ monomeric PRL)/2). GFC monomeric PRL $=$ (amount of monomeric PRL obtained by GFC (\%)/100) $\times$ PRL concentration in the native serum derived from RIA ( $\mu \mathrm{g} / \mathrm{l})$. 
cases, GFC was necessary for definitive confirmation or exclusion of MPRL. This strategy allowed us to significantly reduce the number of time-consuming and expensive GFC required.

Along with the diagnosis of MPRL, it is essential to evaluate the monomeric PRL concentrations in this group, in order to ascertain whether an excess of monomeric PRL is present and determine any potential associations with specific symptoms. Currently, GFC is the gold standard method for separation of the different PRL molecular forms, and so we considered the GFC-yielded monomeric PRL value as the reference. Monomeric PRL, obtained from GFC, was compared with a reference derived from GFC of normal subject sera (1).

The PRL measurement by ECLIA overestimated the monomeric PRL concentration and only weakly correlated with GFC monomeric PRL values. Therefore, its diagnostic value is limited and cannot be used to look for an excess of monomeric PRL in the MPRL group. No PRL assay has an exact specificity for monomeric PRL, even those with low cross-reactivity $(14,15,16,30)$. The heterogeneity of high molecular PRL forms and the resulting difference of crossreactivity with ECLIA, together with their variable amounts in sera, can explain this discrepancy.

The measurement of PRL concentration in the supernatant could also be used to estimate monomeric PRL. However, in the case of MPRL, it was only weakly correlated with GFC-yielded monomeric PRL levels. Thus, this value does not seem to be a reliable estimation of the monomeric PRL concentration in the case of MPRL. It may be speculated that reliability is diminished due to the fact that PEG precipitation is non-specific and a significant, yet highly variable, amount of monomeric PRL is co-precipitated with high molecular forms (25\% on average) (22). Furthermore, the precipitation of high molecular forms of PRL is not perfectly constant. As previously mentioned, IgA-PRL complexes are only partially precipitated. In addition, bPRL may precipitate, at least partially $(22,30)$, but little is known about it. In our study, the serum with predominant bPRL (54\%) had a PRL recovery of $17.0 \%$, which is consistent with bPRL precipitation. Conversely, we did not detect bPRL in the resolubilised precipitate of a serum with 31\% bPRL. Thus, precipitation of bPRL by PEG may vary from one serum to another. Low concentrations of PRL were detected in the supernatants, in MPRL cases. However, the inconsistency between the PRL concentrations in the supernatants and GFC monomeric PRL values did not appear to be inversely related to the PRL levels.

In the 55 female patients of the MPRL group, we compared the classification obtained by using the threshold of PRL in the supernatant, to the classification according to the GFC monomeric PRL levels. Seventeen of the 55 female patients would have been misclassified based on the values of PRL in the supernatant. Sixteen of these would have been misdiagnosed as having an associated excess of monomeric PRL.

In conclusion, PRL recovery after PEG precipitation combined with RIA/ECLIA PRL ratio analysis allowed the definitive exclusion or diagnosis of MPRL, without GFC, in $72.1 \%$ of our hyperprolactinaemic patients. However, in the other $27.9 \%$ of cases, it remained necessary to perform GFC to obtain a definitive diagnosis. GFC was essential in all macroprolactinaemic samples to ascertain whether there existed an associated excess of monomeric PRL.

Declaration of interest

The authors declare that there is no conflict of interest that could be perceived as prejudicing the impartiality of the research reported.

\section{Funding}

This research did not receive any specific grant from any funding agency in the public, commercial or not-for-profit sector.

\section{Acknowledgements}

The authors thank Sophie Kalfon and the hormonology laboratory staff of the Centre de Biologie et de Pathologie Est. They also thank Fabien Subtil for his statistical assistance. The manuscript has been revised for the English by an independent scientific language editing service.

\section{References}

1 Beltran L, Fahie-Wilson MN, McKenna TJ, Kavanagh L \& Smith TP. Serum total prolactin and monomeric prolactin reference intervals determined by precipitation with polyethylene glycol: evaluation and validation on common immunoassay platforms. Clinical Chemistry 200854 1673-1681. (doi:10.1373/clinchem.2008.105312)

2 Fahie-Wilson MN \& Soule SG. Macroprolactinaemia: contribution to hyperprolactinaemia in a district general hospital and evaluation of a screening test based on precipitation with polyethylene glycol. Annals of Clinical Biochemistry 199734 252-258. (doi:10.1177/ 000456329703400305)

3 Whittaker PG, Wilcox T \& Lind T. Maintained fertility in a patient with hyperprolactinemia due to big, big prolactin. Journal of Clinical Endocrinology and Metabolism 198153 863-866. (doi:10.1210/ jcem-53-4-863)

4 Jackson RD, Wortsman J \& Malarkey WB. Characterization of a large molecular weight prolactin in women with idiopathic hyperprolactinemia and normal menses. Journal of Clinical Endocrinology and Metabolism 198561 258-264. (doi:10.1210/jcem-61-2-258)

5 Vallette-Kasic S, Morange-Ramos I, Selim A, Gunz G, Morange S, Enjalbert A, Martin P, Jaquet P \& Brue T. Macroprolactinemia revisited: a study on 106 patients. Journal of Clinical Endocrinology and Metabolism 200287 581-588. (doi:10.1210/jcem.87.2.8272) 
6 Leslie H, Courtney CH, Bell PM, Hadden DR, McCance DR, Ellis PK, Sheridan B \& Atkinson AB. Laboratory and clinical experience in 55 patients with macroprolactinemia identified by a simple polyethylene glycol precipitation method. Journal of Clinical Endocrinology and Metabolism 200186 2743-2746. (doi:10.1210/jcem. 86.6.7521)

7 Leite V, Cosby H, Sobrinho LG, Fresnoza MA, Santos MA \& Friesen HG. Characterization of big, big prolactin in patients with hyperprolactinaemia. Clinical Endocrinology 199237 365-372. (doi:10.1111/j.13652265.1992.tb02340.x)

8 Suliman AM, Smith TP, Gibney J \& McKenna TJ. Frequent misdiagnosis and mismanagement of hyperprolactinemic patients before the introduction of macroprolactin screening: application of a new strict laboratory definition of macroprolactinemia. Clinical Chemistry 2003 49 1504-1509. (doi:10.1373/49.9.1504)

9 Gibney J, Smith TP \& McKenna TJ. The impact on clinical practice of routine screening for macroprolactin. Journal of Clinical Endocrinology and Metabolism 200590 3927-3932. (doi:10.1210/jc.2004-2234)

10 Alfonso A, Rieniets KI \& Vigersky RA. Incidence and clinical significance of elevated macroprolactin levels in patients with hyperprolactinemia. Endocrine Practice 200612 275-280. (doi:10.4158/ EP.12.3.275

11 Hattori N \& Inagaki C. Anti-prolactin (PRL) autoantibodies cause asymptomatic hyperprolactinemia: bioassay and clearance studies of PRL-immunoglobulin G complex. Journal of Clinical Endocrinology and Metabolism 199782 3107-3110.

12 Hattori N, Ikekubo K, Ishihara T, Moridera K, Hino M \& Kurahachi H. A normal ovulatory woman with hyperprolactinemia: presence of anti-prolactin autoantibody and the regulation of prolactin secretion. Acta Endocrinologica 1992126 497-500.

13 Schepper JD, Schiettecatte J, Velkeniers B, Blumenfeld Z, Shteinberg M, Devroey P, Anckaert E, Smitz J, Verdood P, Hooghe R et al. Clinical and biological characterization of macroprolactinemia with and without prolactin-IgG complexes. European Journal of Endocrinology 2003149 201-207. (doi:10.1530/eje.0.1490201)

14 Fahie-Wilson M \& Smith TP. Determination of prolactin: the macroprolactin problem. Best Practice \& Research. Clinical Endocrinology \& Metabolism 201327 725-742. (doi:10.1016/j.beem.2013.07.002)

15 Schneider W, Marcovitz S, Al-Shammari S, Yago S \& Chevalier S. Reactivity of macroprolactin in common automated immunoassays. Clinical Biochemistry 200134 469-473. (doi:10.1016/S00099120(01)00256-9)

16 Smith TP, Suliman AM, Fahie-Wilson MN \& McKenna TJ. Gross variability in the detection of prolactin in sera containing big big prolactin (macroprolactin) by commercial immunoassays. Journal of Clinical Endocrinology and Metabolism 200287 5410-5415. (doi:10.1210/ jc.2001-011943)
17 Fahie-Wilson M. In hyperprolactinemia, testing for macroprolactin is essential. Clinical Chemistry 200349 1434-1436. (doi:10.1373/49.9.1434)

18 Gibney J, Smith TP \& McKenna TJ. Clinical relevance of macroprolactin. Clinical Endocrinology 200562 633-643. (doi:10.1111/j.13652265.2005.02243.x)

19 Vieira JG, Tachibana TT, Obara LH \& Maciel RM. Extensive experience and validation of polyethylene glycol precipitation as a screening method for macroprolactinemia. Clinical Chemistry 1998 44 1758-1759.

20 Olukoga AO \& Kane JW. Macroprolactinaemia: validation and application of the polyethylene glycol precipitation test and clinical characterization of the condition. Clinical Endocrinology $1999 \mathbf{5 1}$ 119-126. (doi:10.1046/j.1365-2265.1999.00757.x)

21 Fahie-Wilson MN. Polyethylene glycol precipitation as a screening method for macroprolactinemia. Clinical Chemistry 199945 436-437.

22 Kavanagh L, McKenna TJ, Fahie-Wilson MN, Gibney J \& Smith TP. Specificity and clinical utility of methods for the detection of macroprolactin. Clinical Chemistry 200652 1366-1372. (doi:10.1373/ clinchem.2005.065854)

23 Sapin R, Gasser F, Fischbach E \& Grucker D. Macroprolactin detection: a new approach. Annales de Biologie Clinique 200058 729-734.

24 Rojat $\mathrm{P}$, Bernard $\mathrm{M}$ \& Coussieu C. Une alternative à la chromatographie pour la détection de la macroprolactine: calcul du rapport de deux immunodosages de la prolactine: Immulite $2000 ®$ et Kryptor®. Immuno-analyse \& Biologie Spécialisée 200722 115-119. (doi:10.1016/ j.immbio.2007.02.001)

25 McCudden CR, Sharpless JL \& Grenache DG. Comparison of multiple methods for identification of hyperprolactinemia in the presence of macroprolactin. Clinica Chimica Acta 2010411 155-160. (doi:10.1016/ j.cca.2009.10.020)

26 Smith TP \& Fahie-Wilson MN. Reporting of post-PEG prolactin concentrations: time to change. Clinical Chemistry 201056 484-485. (doi:10.1373/clinchem.2009.135210)

27 Sassolas G, Chatelain P, Cohen R, Boissel JP, Laporte S, Galleyrand J, Claustrat B, Elmcharfi A, Chayvialle JA, Cohen $\mathrm{H}$ et al. Effects of human pancreatic tumor growth hormone-releasing hormone (hpGRH144-NH2) on immunoreactive and bioactive plasma growth hormone in normal young men. Journal of Clinical Endocrinology and Metabolism 198459 705-709. (doi:10.1210/jcem-59-4-705)

28 Champier J, Claustrat B, Harthe C, Abd-Elouahab B \& Sassolas G. Thiol reduction of a glycosylated human plasma prolactin variant generates a cleaved fragment. Neuro Endocrinology Letters 199113 261-268.

29 Jamaluddin FA, Sthaneshwar P, Hussein Z, Othman N \& Chan SP. Importance of screening for macroprolactin in all hyperprolactinaemic sera. Malaysian Journal of Pathology 201335 59-63.

30 Ellis MJ, Livesey JH \& Soule SG. Macroprolactin, big-prolactin and potential effects on the misdiagnosis of hyperprolactinemia using the Beckman Coulter Access Prolactin assay. Clinical Biochemistry 200639 1028-1034. (doi:10.1016/j.clinbiochem.2006.06.003)

Received 6 December 2014

Revised version received 26 February 2015

Accepted 9 March 2015 